\title{
Constraints on the Nucleosynthesis of Refractory Nuclides in Galactic Cosmic Rays
}

\author{
M. E. Wiedenbeck*, W. R. Binns ${ }^{\dagger}$, E. R. Christian**, A. C. Cummings ${ }^{\ddagger}$, \\ A. J. Davis ${ }^{\ddagger}$, J. S. George ${ }^{\ddagger}$, P. L. Hink ${ }^{\dagger}$, M. H. Israel ${ }^{\dagger}$, R. A. Leske ${ }^{\ddagger}$, \\ R. A. Mewaldt ${ }^{\ddagger}$ E. C. Stone ${ }^{\ddagger}$, T. T. von Rosenvinge ${ }^{* *}$ and N. E. Yanasak ${ }^{\ddagger}$ \\ ${ }^{*}$ Jet Propulsion Laboratory, California Institute of Technology, Pasadena, CA 91109 USA \\ Washington University, St. Louis, MO 63130 USA \\ ${ }^{* *}$ NASA/Goddard Space Flight Center, Greenbelt, MD 20771 USA \\ ${ }^{\ddagger}$ California Institute of Technology, Pasadena, CA 91125 USA
}

\begin{abstract}
Abundances of the isotopes of the refractory elements $\mathrm{Ca}, \mathrm{Fe}, \mathrm{Co}$, and $\mathrm{Ni}$ in the galactic cosmic-ray source are compared with corresponding abundances in solar-system matter. For the 12 nuclides considered, relative abundances agree to within a factor of 2, and typically within 20-30\%. In addition, comparisons of cosmic-ray abundances with model calculations of supernova yields are used to argue that cosmic rays contain contributions from stars with a broad range of masses. Based on these and other results we suggest that cosmic rays probably represent a sample of contemporary interstellar matter, at least for refractory species.
\end{abstract}

\section{INTRODUCTION}

Over the past decade considerable progress has been made in precisely determining the isotopic composition of cosmic rays. Instruments on the Advanced Composition Explorer (ACE) and Ulysses missions have succeeded in resolving cosmic-ray isotopes up through the iron group, and the large geometrical acceptance of the Cosmic-Ray Isotope Spectrometer (CRIS) on ACE has made possible abundance measurements of very rare nuclides, including the first isotope measurements in the region $Z>28$. Among the $80+$ individual nuclides for which abundances have been measured in cosmic rays arriving near Earth, approximately one third are dominated by primary nuclei, which are accelerated in the Galaxy and have only relatively small contributions from secondary particles produced by fragmentation of heavier species during passage through the interstellar medium. For this subset of the observed nuclides it is possible to infer the abundances in the source material from which the particles were accelerated.

The increase in the number and precision of nuclidic abundance determinations in the cosmic-ray source is providing a new tool for studies of astrophysics in our Galaxy. These data are yielding information on the stellar sources in which the cosmic-ray material was synthesized. In addition, they are providing a detailed determination of the isotopic make-up of a sample of material which may be representative of the composition of contemporary interstellar matter.

\section{OBSERVATIONS}

The CRIS instrument [1] measures the charge, mass, and energy of detected nuclei using multiple measurements of $d E / d x$ versus total energy made with stacks of silicon solid state detectors. In addition, particle trajectories are measured in a scintillating optical-fiber hodoscope to enable corrections for particle pathlengths through the silicon detectors. The geometrical factor of the instrument, $\sim 250 \mathrm{~cm}^{2} \mathrm{sr}$, is large enough to obtain statistically significant measurements of even relatively rare nuclides. Figure 1 shows mass histograms obtained from CRIS for elements from $\mathrm{Li}$ through $\mathrm{Cu}$. The events included in these histograms were selected to have ranges long enough to penetrate into at least the third silicon detector in the stack, yielding multiple mass measurements which are required to be consistent. In addition, to obtain a data set with particularly high resolution the events were required to have trajectories within $25^{\circ}$ of the normal to the detector surfaces. In this data set, which contains approximately $30 \%$ of the particles collected, essentially all the stable and long-lived isotopes up through the iron group are resolved. For nuclides separated by $2 \mathrm{amu}$, including the rare isotopes ${ }^{46} \mathrm{Ca},{ }^{48} \mathrm{Ca},{ }^{57} \mathrm{Co},{ }^{59} \mathrm{Co},{ }^{64} \mathrm{Ni},{ }^{63} \mathrm{Cu}$, and

CP598, Solar and Galactic Composition, edited by R. F. Wimmer-Schweingruber

(C) 2001 American Institute of Physics 0-7354-0042-3/01/\$18.00 


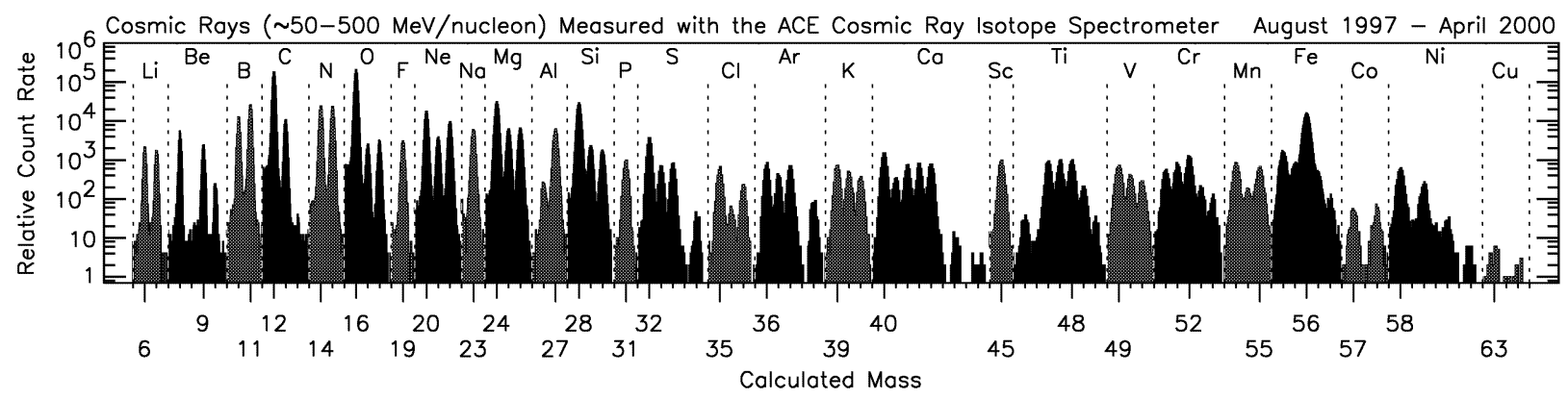

FIGURE 1. Cosmic-ray mass histograms from the Cosmic-Ray Isotope Spectrometer on ACE. Even and odd- $Z$ elements are shaded different colors for clarity. Ticks along the abscissa indicate integral masses, with one tick labeled for each element. Note the logarithmic ordinate-the dynamic range in abundance shown is $>10^{5}$.

${ }^{65} \mathrm{Cu}$, particles with more extreme angles of incidence can also be used in the analysis, improving the statistical accuracy for these species.

The isotopic compositions of individual elements are derived from the relative areas of the isotope peaks in the mass histograms by applying relatively small corrections for differences in energy intervals and nuclear interaction probabilities in the instrument. A different analysis procedure is used to obtain the relative abundances of the elements, since energy intervals differ considerably for elements when charges are not closely spaced. The energy spectrum of each element is derived from the measured count rates and live times, combined with calculated energy intervals, geometrical factors, interaction loss probabilities, and detection efficiencies. Spectral shapes are generally similar, particularly for elements dominated by primary cosmic rays. Flux values are interpolated to a common energy per nucleon within the measurement intervals for the elements being compared. For the elements $\mathrm{Ca}, \mathrm{Fe}, \mathrm{Co}$, and Ni considered here, an energy of $300 \mathrm{MeV} /$ nucleon was used. The data analysis and the derived cosmic-ray composition near Earth are discussed more thoroughly in [2].

\section{COSMIC-RAY SOURCE ABUNDANCES}

In order to investigate the nucleosynthesis of cosmic-ray source material we have selected nuclides which 1 ) have relatively small contributions from secondary cosmic rays produced by spallation reactions in the interstellar medium and 2) are thought to have experienced minimal fractionation relative to one another during cosmic-ray injection and acceleration. The first requirement is satisfied by $\mathrm{Fe}$ and species slightly heavier than $\mathrm{Fe}$, since abundances in this region are rapidly decreasing with increasing mass so that there is relatively little material that can fragment into a nuclide of interest. In addition, the extreme isotopes of calcium, ${ }^{40} \mathrm{Ca}$ and ${ }^{48} \mathrm{Ca}$, have small secondary contributions. These doubly-magic nuclei have mass-to-charge ratios considerably different from that of ${ }^{56} \mathrm{Fe}$, the most abundant nuclide that could fragment into them. The large change of $A / Z$ required to produce these isotopes results in small production cross sections.

To avoid the complications of needing to correct for fractionation, our study focused on elements with low first ionization potential (FIP) and high condensation temperatures (low volatility). As has been extensively discussed (e.g, [3] and references therein), the ratio of cosmic-ray source elemental abundances to corresponding abundances in solar-system material are relatively constant for elements with FIP values less than about $9 \mathrm{eV}$, whereas higher-FIP elements are significantly depleted in cosmic rays. The physical mechanism for this fractionation is not conclusively established. Processes controlled by volatility rather than FIP have been proposed [3] and the general correlation of these two parameters makes it difficult to distinguish between these possibilities. Elements that are particularly volatile in spite of low FIP values have been avoided in the present study because of this uncertainty about how the elemental fractionation occurs. The elements $\mathrm{Ca}, \mathrm{Fe}, \mathrm{Co}$, and $\mathrm{Ni}$ have both low FIP and low volatility, and thus should experience little, if any, fractionation relative to one another.

Source abundances can be reliably derived for the nuclides we are considering by taking advantage of the large number of isotopes with masses $40<A<56$ that are dominated by secondary fragmentation products. Using a simple leaky-box model of the interstellar propagation of cosmic rays and adjusting the escape length to reproduce the observed abundances of such secondaries we obtain estimates of the secondary contributions (generally small) to the nuclides for which source abundances are to be derived. These calculations are described in detail in [2].

The filled circles in Fig. 2 show the source composition derived from the CRIS observations, both as abundances relative to ${ }^{56} \mathrm{Fe} \equiv 1$ and as ratios to solar system abundances [4]. In addition, source abundances re- 


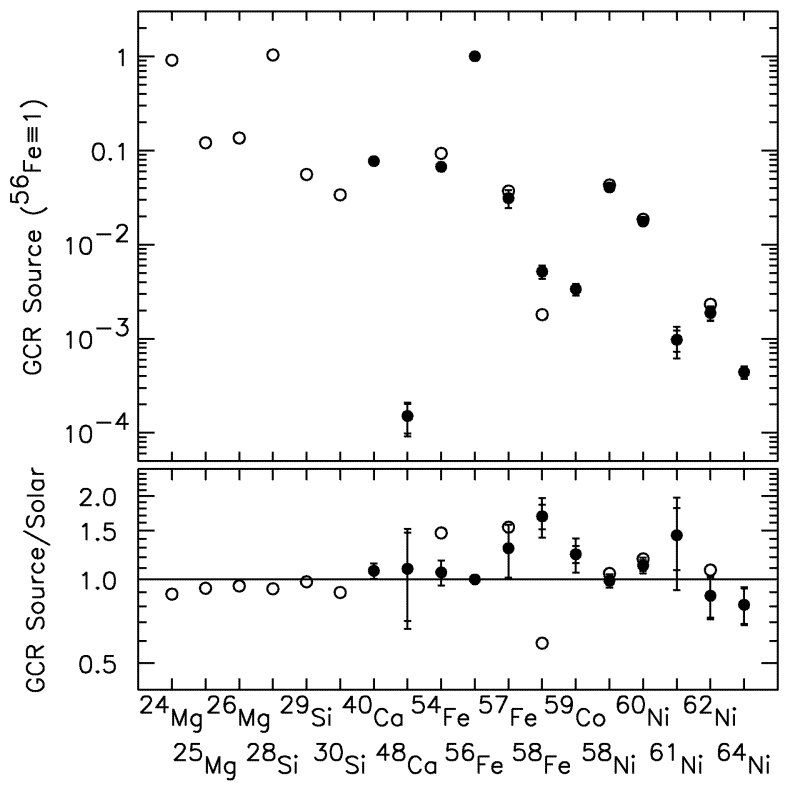

FIGURE 2. Cosmic-ray source composition from ACE (filled circles) and Ulysses (open circles). Cosmic-ray source abundances for the set of refractory nuclides shown range over nearly 4 orders of magnitude (upper panel), but in all cases are consistent with solar-system abundances [4] to within better than a factor of 2 (lower panel). For the ACE data the inner error bars represent statistical uncertainties while the outer error bars also incorporate estimates of systematic and propagation uncertainties [2]. Ulysses uncertainties (not shown) are discussed in $[5,6]$.

ported from Ulysses for the isotopes of $\mathrm{Mg}, \mathrm{Si}, \mathrm{Fe}$, and $\mathrm{Ni}[5,6]$ are plotted as the open circles. For normalizing the Ulysses abundances to ${ }^{56} \mathrm{Fe}$ we have used the source elemental ratios derived from HEAO-C2 observations [7]. In the cases where abundance measurements are available from both ACE and Ulysses, the agreement is generally quite good. Possible explanations of the most significant differences (for ${ }^{54} \mathrm{Fe}$ and ${ }^{58} \mathrm{Fe}$ ) have been discussed in [2,8]. CRIS also measures the isotopes of the refractory elements $\mathrm{Mg}$ and $\mathrm{Si}$ [2], but we have not yet performed propagation calculations that account for secondary-nuclide abundances over the full charge range $9 \leq Z \leq 28$ with the accuracy needed to take full advantage of the small statistical uncertainties in the CRIS observations.

The nuclides we are considering have absolute abundances ranging over a factor $\sim 10^{4}$. However, the abundance distribution is consistent with that found in solar system material to better than a factor of 2 for all of these nuclides, and typically within $\sim 20-30 \%$. This similarity is particularly striking in view of the fact that the various isotopes represented in Fig. 2 are thought to originate in a diverse range of stellar environments. Table 1 summarizes the nucleosynthesis processes and sites believed to
Table 1.

Major Nuclesynthetic Contributions ${ }^{a}$

\begin{tabular}{llll}
\hline Nucl. & \multicolumn{1}{c}{ Origin $^{b}$} & Nucl. & \multicolumn{1}{c}{ Origin $^{b}$} \\
\hline${ }^{24} \mathrm{Mg}$ & $\mathrm{C}, \mathrm{Ne}$ & ${ }^{56} \mathrm{Fe}$ & $\mathrm{xSi}, \mathrm{Ia}$ \\
${ }^{25} \mathrm{Mg}$ & $\mathrm{C}, \mathrm{Ne}, \mathrm{He}(\mathrm{s})$ & ${ }^{57} \mathrm{Fe}$ & $\mathrm{xSi}, \mathrm{Ia}$ \\
${ }^{26} \mathrm{Mg}$ & $\mathrm{C}, \mathrm{Ne}, \mathrm{He}(\mathrm{s})$ & ${ }^{58} \mathrm{Fe}$ & $\mathrm{He}(\mathrm{s}), \mathrm{nse}-\mathrm{Ia}-\mathrm{MCh}$ \\
${ }^{28} \mathrm{Si}$ & $\mathrm{xO}, \mathrm{O}$ & ${ }^{59} \mathrm{Co}$ & $\mathrm{He}(\mathrm{s}), \alpha, \mathrm{Ia}, \nu$ \\
${ }^{29} \mathrm{Si} \quad \mathrm{Ne}, \mathrm{xNe}$ & ${ }^{58} \mathrm{Ni}$ & $\alpha, \mathrm{Ia}$ \\
${ }^{30} \mathrm{Si}$ & $\mathrm{Ne}, \mathrm{xNe}$ & ${ }^{60} \mathrm{Ni}$ & $\alpha, \mathrm{He}(\mathrm{s})$ \\
${ }^{40} \mathrm{Ca} \quad \mathrm{xO}, \mathrm{O}$ & ${ }^{61} \mathrm{Ni}$ & $\alpha, \mathrm{Ia}-\mathrm{det}, \mathrm{He}(\mathrm{s})$ \\
${ }^{48} \mathrm{Ca}$ & $\mathrm{nse}-\mathrm{Ia}-\mathrm{MCh}$ & ${ }^{62} \mathrm{Ni}$ & $\alpha, \mathrm{He}(\mathrm{s})$ \\
${ }^{54} \mathrm{Fe} \quad \mathrm{Ia}, \mathrm{xSi}$ & ${ }^{64} \mathrm{Ni}$ & $\mathrm{He}(\mathrm{s})$ \\
\hline${ }^{a}$ from Woosley \& Weaver (1995) \\
${ }^{b} \mathrm{Abbreviations} \mathrm{for} \mathrm{processes} \mathrm{\&} \mathrm{sites} \mathrm{are} \mathrm{as} \mathrm{fol-}$ \\
lows: C, O, Ne: quiescent burning of these ele- \\
ments; xO, xNe, xSi: explosive burning of these \\
elements; He(s): s-process during core/shell He \\
burning; $\alpha$ : $\alpha$-rich freeze out during SN II explo- \\
sion; Ia: SN Ia igniting at Chandrasehkar mass; \\
nse-Ia-MCh: inner n-rich regions of SN Ia ignit- \\
ing at Chandrasehkar mass; Ia-det: SN Ia from \\
detonation at less than Chandrasehkar-mass; $\nu$ : \\
neutrino process.
\end{tabular}

be major contributors to the individual nuclides found in solar system material [9].

From the similarity between cosmic-ray source and solar-system compositions one expects the same processes to be important contributors to cosmic rays. However, the relative contributions of various nucleosynthetic processes to solar-system and galactic cosmic-ray (GCR) matter are not "universal". For example, in our neighboring galaxies, the Large and Small Magellanic Clouds, it has been found [10] that the contributions from Type Ia supernovae are significantly greater than in the Milky Way. Thus one can expect to obtain new insights into the origin of cosmic rays and/or solar system material by understanding the close correspondence of the compositions of these two populations.

\section{SUPERNOVA CONTRIBUTIONS TO COSMIC RAYS}

In massive stars, the production of most of the iron-group material occurs during the final stages of hydrostatic stellar evolution. The synthesis of these nuclides, for which the binding energy per nucleon is maximum, marks the exhaustion of a star's nuclear fuel. The ensuing collapse of the stellar core and its explosive rebound leads to further alteration of abundances. Detailed numerical calculations have been carried out by two groups $[9,11]$ to determine the composition of the material returned to the interstellar medium in such core-collapse supernovae (SN II) arising from a range of progenitor star masses.

For many nuclides very similar yields are reported 
from these two investigations. However, for several nuclides of interest for this study there are important differences. We have chosen to adopt the results from [9], based on a detailed evaluation of the difference between the two calculational approaches [12]. It must also be recognized that massive-star models have not yet successfully accounted for the supernova core bounce that drives the explosive processing and ejection of material. Instead explosions are artificially put into the calculations, constrained by observed properties of SN II. Until the physics of the explosions is adequately understood, there remains the possibility of large errors in predicted yields, particularly in the iron-dominated stellar layers close to the "mass cut" separating the matter that is ejected from that which collapses into the remnant.

Important contributions of iron-group nuclides also come from Type Ia supernovae (SN Ia), which are thought to arise from the accretion of matter onto white dwarf stars in binary systems and the subsequent nuclear deflagration/detonation. Numerical calculations of these yields have also been reported [13]. However, the detailed physics of the SN Ia explosion are not yet firmly established, and various scenarios that have been considered lead to significantly different nucleosynthesis yields. For this study we have adopted the yields of the widely-used "W7" model (see [13] and references therein) as characteristic of the yields of low-mass $\left(M<8 M_{\odot}\right)$ stars that evolve into white dwarves and ultimately explode as SN Ia.

To investigate the contributions of various supernova types to the cosmic-ray source, we have compared the cosmic-ray source abundances derived from the CRIS data with the available supernova yield calculations. These comparisons are shown in Fig. 3, where the cosmic-ray source abundances are plotted as the horizontal solid line with dotted lines indicating the $\pm 1 \sigma$ uncertainty band. The SN II yields from various mass progenitor stars are plotted as the open symbols and the $\mathrm{SN}$ Ia yields as the filled symbol. For some ratios the calculations can reproduce the cosmic-ray abundances for a range of stellar masses. However, no single mass can account for most of the nuclides shown. Thus, for example, stars more massive than $\sim 15 M_{\odot}$ can account for the observed ${ }^{58} \mathrm{Ni} /{ }^{56} \mathrm{Fe}$, whereas to account for ${ }^{64} \mathrm{Ni} /{ }^{56} \mathrm{Fe}$ requires stars less massive than this value. For some species, most notably ${ }^{48} \mathrm{Ca}$, none of these supernova models can adequately reproduce the observations.

To obtain quantitative limits on the amount of material that supernovae of various progenitor masses could have contributed to the cosmic-ray source, we have considered the effect of mixing material with the composition given by each of the supernova models into a pool of material from other sources. The ratio, by mass, of two nuclides $X$ and $Y$ in the mixed material is

$$
\begin{aligned}
(X / Y)_{m} & =\frac{M_{i}(Y) \cdot(X / Y)_{i}+M_{p}(Y) \cdot(X / Y)_{p}}{M_{i}(Y)+M_{p}(Y)} \\
& =f \cdot(X / Y)_{i}+(1-f) \cdot(X / Y)_{p} .
\end{aligned}
$$

Here the subscripts refer to the resulting mixed material, $m$, the material from a particular supernova type, $i$, and material from the pool, $p$, into which the supernova material is being mixed. $M_{i}(Y)$ and $M_{p}(Y)$ are the masses of nuclide $Y$ in the two original populations, while $(X / Y)_{i}$ and $(X / Y)_{p}$ are the abundance ratios by mass. The symbol $f \equiv M_{i}(Y) /\left(M_{i}(Y)+M_{p}(Y)\right)$ denotes the fraction of nuclide $Y$ in the mix that came from sources of type $i$.

If $(X / Y)_{i}$ is larger than $(X / Y)_{m}$ then there is a maximum fraction of material from supernova type $i$ that could have been included in the mix without producing a value of $(X / Y)_{m}$ exceeding that found in the cosmic-ray source. This maximum is realized if nuclide $X$ is absent in the background material, $(X / Y)_{p}=0$. The resulting upper limit on the fraction of nuclide $Y$ from source $i$ is given by $f_{\max }=(X / Y)_{m} /(X / Y)_{i}$.

It should be noted that this approach is providing an upper limit on the fractional contribution of a particular supernova type to the overall abundance of $Y$, the nuclide in the denominator of the ratio being considered. To convert this value into a limit on the fraction of the total mass contributed by the model (including all nuclides, not just $X$ and $Y$ ), one would need to know the fraction by mass of nuclide $Y$ in the material into which the supernova ejecta are being mixed-this would require an additional assumption about the nature of that material.

For illustration, we consider the ratio ${ }^{58} \mathrm{Ni} /{ }^{56} \mathrm{Fe}$. Since the value of this abundance ratio expected from a supernova with a $11 M_{\odot}$ progenitor is approximately 5 times the value in the cosmic-ray source (Fig. 3), this source could have contributed no more than $\sim 20 \%$ of the cosmic-ray ${ }^{56} \mathrm{Fe}$. Figure 4 shows the upper limits derived in this way for each stellar model. The limits are most stringent for those models that produce ${ }^{58} \mathrm{Ni} /{ }^{56} \mathrm{Fe}$ with the largest excesses over the cosmic-ray value. When the cosmic-ray ${ }^{58} \mathrm{Ni} /{ }^{56} \mathrm{Fe}$ ratio is greater than that obtained from a given model, no constraint on the contribution of that model to the ${ }^{56} \mathrm{Fe}$ is obtained. (But one could obtain a limit on the fractional contribution to ${ }^{58} \mathrm{Ni}$ by considering the reciprocal ratio.)

Similar analyses can be done using the measured ratios of the other isotopes relative to ${ }^{56} \mathrm{Fe}$. From each isotope one obtains a set of upper limits on the amounts of ${ }^{56} \mathrm{Fe}$ that could have been contributed by the various mass stars. Applying the limits from all the isotopes together one obtains a more stringent set of upper limits, with the amount of material from each stellar mass constrained by the most restrictive of the individual limits. Figure 5 shows (open circles) the upper limits obtained 

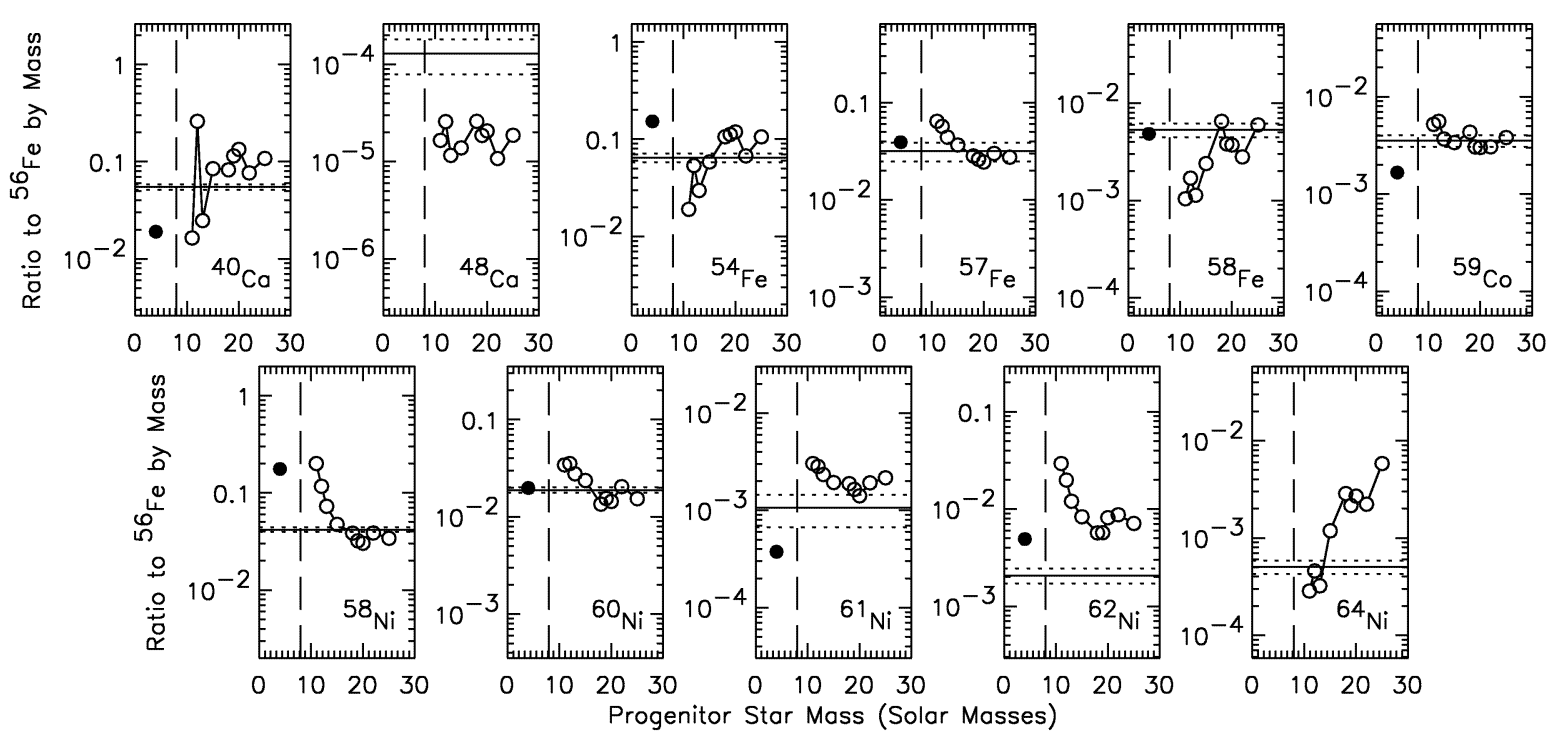

FIGURE 3. Comparison of GCR source abundance ratios with calculated values for yields of supernovae from various masses of progenitor star (filled circle: SN Ia [13]; open circles: SN II [9]). Cosmic-ray source values and their $\pm 1 \sigma$ uncertainties are shown by the horizontal lines. The approximate mass boundary between low-mass stars that lead to SN Ia and high-mass stars that lead to SN II is shown by the dashed lines.

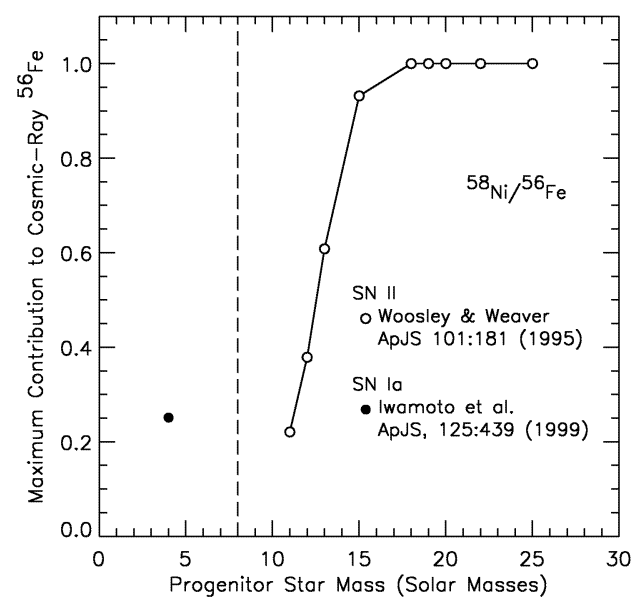

FIGURE 4. Limits on the maximum fraction of the GCR ${ }^{56} \mathrm{Fe}$ that could have originated from supernovae of various mass progenitor stars based on the observed ${ }^{58} \mathrm{Ni} /{ }^{56} \mathrm{Fe}$ ratio.

by requiring that none of the isotope ratios relative to ${ }^{56} \mathrm{Fe}$ exceed their cosmic-ray values in the overall mix of material. Of course, if there are significant errors in the model calculations for a particular isotope, any upper limit driven by this isotope would be invalidated. Figure 5 also shows the effect of allowing the violation of the most restrictive one or two individual isotope limits (open squares and triangles, respectively).

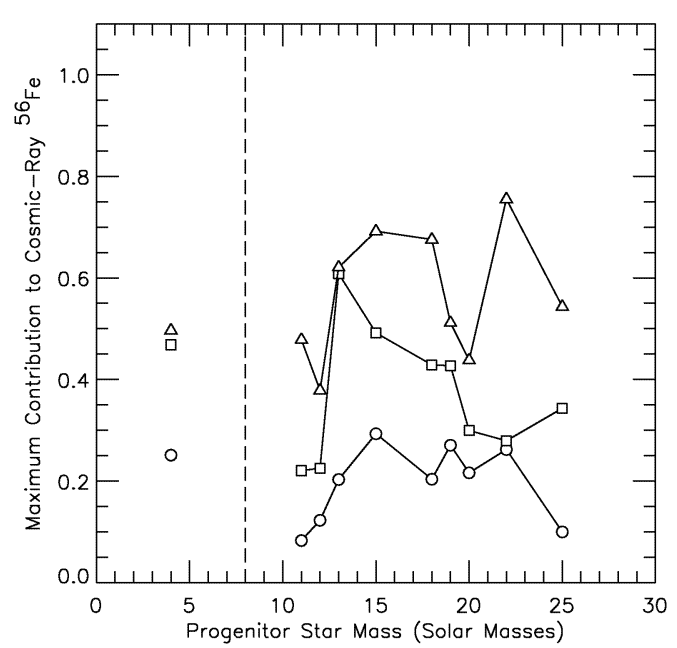

FIGURE 5. Limits on the maximum fraction of the GCR ${ }^{56} \mathrm{Fe}$ that could have originated from supernovae of various mass progenitor stars given the derived GCR source abundances for all the $\mathrm{Ca}, \mathrm{Fe}, \mathrm{Co}$, and $\mathrm{Ni}$ isotopes studied. The calculated upper limits take into account the $1 \sigma$ uncertainty in the cosmic-ray source value, but no uncertainties in the nucleosynthesis models. Circles, squares, and triangles indicate the limits obtained by disregarding the 0,1 , or 2 most restrictive constraints, respectively.

\section{DISCUSSION}

The most notable feature of the combined upper limits on the contributions of various mass stars to cosmic-ray 
source material (Fig. 5) is the fact that none of the star types considered could be dominating the production of this material. Since the abundance yields are a relatively smooth function of the star's mass (Fig. 3), we conclude that the limits we derive apply not just to the discrete masses for which yield calculations are available, but also for ranges of stellar masses around these values.

The essential constraints we have on the nature of the cosmic-ray source are its compositional similarity to solar-system material and the fact that it apparently contains significant contributions from a wide range of stellar masses. One would expect such features if the cosmic rays were being accelerated out of the general pool of interstellar matter, since the solar system condensed from such a pool, albeit $\sim 4.5$ Gyr earlier. Furthermore, the initial mass function (IMF) in the Galaxy is known to have a relatively smooth dependence on stellar mass, and the Galaxy is old enough that stellar birth and death rates should be similar provided that the IMF has not changed greatly over the past æon.

The suggestion that supernova material gets well mixed with interstellar matter before cosmic-ray acceleration occurs is supported by the observation that a time $\gtrsim 10^{5} \mathrm{yr}$ elapses between nucleosynthesis and acceleration [14], since this time is significantly longer than that required for a supernova remnant to dissipate its energy as it expands into matter of average interstellar density.

There remain uncertainties related to possible spatial and temporal effects that could produce differences between the material being sampled by cosmic rays and that found in the solar system. For example, the higher density of stars toward the galactic center is thought to be causing a more rapid evolution of the composition of the ISM there. Whether this should affect the cosmic-ray composition observed near Earth depends on poorly constrained aspects of cosmic-ray transport in the Galaxy.

It would also be of considerable interest to determine whether models of galactic chemical evolution (GCE) can account for the small amount of evolution of interstellar abundances suggested by our comparison between solar-system and cosmic-ray material. Unfortunately, GCE studies generally have not been extended to cover the time between solar-system formation and the present, concentrating instead on evolution up to this time. Furthermore, reports of GCE results have often not included the abundances of individual nuclides that would be needed to take advantage of observations such as those shown in Fig. 2.

For reasons discussed above, the work on cosmic-ray source composition presented here has concentrated on refractory nuclides. A model accounting for the origin of cosmic-ray source material must ultimately also address the abundances of volatile species. The isotopic composition of the element neon remains a puzzle: the ratio ${ }^{22} \mathrm{Ne} /{ }^{20} \mathrm{Ne}$ in the cosmic-ray source exceeds the value found in the Sun and inferred for the contemporary very local interstellar medium by a factor $\sim 5$ ([15] and references therein), so it appears difficult to fit this element into the scenario we have discussed for the refractories. A widely-discussed solution to this problem invokes special sources (specifically, Wolf-Rayet stars) that would dominate the production of ${ }^{22} \mathrm{Ne}$ in cosmic rays while not greatly affecting the overall amount of this isotope being contributed to the interstellar medium. It will be important to take advantage of the detailed and precise cosmicray source composition data now becoming available to search for smaller isotopic differences predicted to be associated with the ${ }^{22} \mathrm{Ne}$ anomaly.

\section{ACKNOWLEDGMENTS}

This research was supported by the National Aeronautics and Space Administration at the California Institute of Technology (under grant NAG5-6912), the Jet Propulsion Laboratory, NASA's Goddard Space Flight Center, and Washington University.

\section{REFERENCES}

1. Stone, E.C. et al. 1998, Space Sci. Rev. 86, 285

2. Wiedenbeck, M.E. et al. 2001, Space Sci. Rev., in press

3. Meyer, J.-P., Drury, L. O'C., \& Ellison, D. C. 1997, Astroph. J. 487, 182

4. Anders, E. \& Grevesse, N. 1989, Geoch. Cosmoch. Acta 53, 197

5. Connell, J.J. \& Simpson, J.A. 1997, Astroph. J. Letters 475, L61

6. Connell, J.J. \& Simpson, J.A. 1997, Proc. 25th Internat. Cosmic Ray Conf. (Durban) 3, 381

7. Engelmann, J.J., Ferrando, P., Soutoul, A., Goret, P., Juliusson, E., Koch-Miramond, L., Lund, N., Masse, P., Peters, B., Petrou, N., \& Rasmussen, I.L. 1990, Astron. Astroph. 233, 96

8. Wiedenbeck, M.E. et al. 2001, Adv. Space Res., 27, 773

9. Woosley, S.E. \& Weaver, T.A. 1995, Astroph. J. Suppl. 101,181

10. Tsujimoto, T., Nomoto, K., Yoshii, Y., Hashimoto, M., Yanagida, S., \& Thielemann, F.-K. 1995, Mon. Not. Royal Astron. Soc. 277, 945

11. Thielemann, F.-K., Nomoto, K., \& Hashimoto, M. 1996, Astroph. J. 460, 408

12. Hoffmann, R.D., Woosley, S.E., Weaver, T.A., Rauscher, T., \& Thielemann, F.-K. 1999, Astroph. J. 521, 735

13. Iwamoto, K., Brachwitz, F., Nomoto, K., Kishimoto, N., Umeda., H., Hix, W.R., \& Thielemann, F.-K. 1999, Astroph. J. Suppl. 125, 439

14. Wiedenbeck, M.E. 1999, Astroph. J. Letters 523, L61

15. Binns, W.R. et al. 2001, Joint SOHO-ACE Workshop, this volume (AIP: New York) submitted 